\title{
Article \\ Upregulated Guanine Deaminase Is Involved in Hyperpigmentation of Seborrheic Keratosis via Uric Acid Release
}

\author{
Kyung Ah Cheong ${ }^{1}$, In Sup Kil ${ }^{2}$, Hyuk Wan Ko ${ }^{3}$ and Ai-Young Lee ${ }^{1, *}$ \\ 1 Department of Dermatology, Dongguk University Ilsan Hospital, 814 Siksa-dong, Ilsandong-gu, \\ Goyang-si 410-773, Gyeonggi-do, Korea; bionase@hanmail.net \\ 2 Basic Research \& Innovation Division, Amorepacific Corporation R\&D Center, \\ Yongin-si 446-729, Gyeonggi-do, Korea; iskil@amorepacific.com \\ 3 Department of Biochemistry, College of Life Science and Biotechnology, Yonsei University, Seoul 03722, Korea \\ kohw@yonsei.ac.kr \\ * Correspondence: lay5604@naver.com; Tel.: +82-3-1961-7250
}

Citation: Cheong, K.A.; Kil, I.S.; Ko, H.W.; Lee, A.-Y. Upregulated Guanine Deaminase Is Involved in Hyperpigmentation of Seborrheic Keratosis via Uric Acid Release. Int. J. Mol. Sci. 2021, 22, 12501. https:// doi.org/10.3390/ijms222212501

Academic Editor: Genji Imokawa

Received: 13 August 2021

Accepted: 16 November 2021

Published: 19 November 2021

Publisher's Note: MDPI stays neutral with regard to jurisdictional claims in published maps and institutional affiliations.

Copyright: (c) 2021 by the authors. Licensee MDPI, Basel, Switzerland. This article is an open access article distributed under the terms and conditions of the Creative Commons Attribution (CC BY) license (https:/ / creativecommons.org/licenses/by/ $4.0 /)$.

\begin{abstract}
Seborrheic keratosis, which is a benign tumor composed of epidermal keratinocytes, develops common in the elderly. Uric acid generated by upregulated guanine deaminase (GDA) has been identified to cause UV-induced keratinocyte senescence in seborrheic keratosis. Seborrheic keratosis is also frequently pigmented. Growing evidences indicate that hyperuricemia is a risk factor of acanthosis nigricans, an acquired skin hyperpigmentation. The objective of this study was to investigate role of GDA and its metabolic end product, uric acid, in hyperpigmentation of patients with seborrheic keratosis using their lesional and non-lesional skin specimen sets and cultured primary human epidermal keratinocytes with or without GDA overexpression or uric acid treatment. GDA-overexpressing keratinocytes or their conditioned media containing uric acid increased expression levels of MITF and tyrosinase in melanocytes. Uric acid released from keratinocytes was facilitated by ABCG2 transporter with the help of PDZK1 interaction. Released uric acid was taken by URAT1 transporter in melanocytes, stimulating melanogenesis through p38 MAPK activation. Overall, GDA upregulation in seborrheic keratosis plays a role in melanogenesis via its metabolic end product uric acid, suggesting that seborrheic keratosis as an example of hyperpigmentation associated with photoaging.
\end{abstract}

Keywords: GDA; photoaging; hyperpigmentation; seborrheic keratosis; uric acid

\section{Introduction}

Seborrheic keratosis is a common benign skin tumor characterized by keratinocyte proliferation. Its predilection sites are sun-exposed areas and its affected age group is the elderly [1,2], suggesting the role of photoaging in the development of seborrheic keratosis $[3,4]$. We have recently found that guanine deaminase (GDA) expression in keratinocytes induced by repeated ultraviolet (UV) exposure is involved in UV-induced keratinocyte senescence in seborrheic keratosis via uric acid, a guanine metabolite [5], supporting a causative role of photoaging in seborrheic keratosis. Sunlight can stimulate melanin production to protect skin against harmful effects of UV radiation. Tyrosinase and tyrosinaserelated proteins (TRPs) are key enzymes for melanogenesis. Genes encoding tyrosinase and TRPs contain common transcription starting sites, particularly micropthalmia-associated transcription factor (MITF) binding sites. MITF plays a fundamental role in the transcriptional regulation of melanogenesis [6]. Seborrheic keratosis frequently presents as sharply demarcated light brown to black papules due to great amount of melanin in its keratinocytes. It is considered as one of common facial pigmentary disorders of greatest concern along with melasma [7]. Although the role of uric acid in skin hyperpigmentation is unknown, hyperuricemia is well-known to be associated with metabolic syndrome [8-12]. 
Among cutaneous hyperpigmentations due to increased melanin, acanthosis nigricans characterized by velvety brownish-black thickened hyperpigmentation $[13,14]$ is a representative disorder associated with metabolic syndrome. Growing evidence indicates that hyperuricemia is an important risk factor for acanthosis nigricans $[15,16]$. We assumed that uric acid generated by GDA could contribute to hyperpigmentation in seborrheic keratosis. Thus, the objective of this study was to examine whether and how GDA and its metabolic end product, uric acid, were involved in melanogenesis and skin hyperpigmentation in patients of with seborrheic keratosis.

\section{Results}

\subsection{GDA Upregulation in Keratinocytes Stimulates Melanogenesis by Releasing Uric Acid}

GDA mRNA and protein have been detected in keratinocytes, but not in melanocytes [5]. Thus, the role of upregulated GDA in melanogenesis was examined in primary cultured adult normal human keratinocytes with or without GDA overexpression cocultured with primary cultured adult normal human melanocytes. Levels of tyrosinase mRNA and protein were increased in normal human melanocytes cocultured with GDA-overexpressing keratinocytes (Figure 1a). Levels of tyrosinase and MITF proteins were also elevated in melanocyte monocultures treated with supernatant obtained from GDA-overexpressing keratinocytes (Figure 1b). GDA concentrations were increased in conditioned media from $G D A$-overexpressing keratinocytes without showing a significant effect on cell survival and cytotoxicity (Figure 1c). However, recombinant GDA did not increase tyrosinase protein levels in melanocytes (Figure 1d). Concentrations of uric acid, a metabolic end product of $G D A$, measured for 2 days in culture supernatants after $G D A$ overexpression were increased with the lapse of time (Figure 1e). Exogenous uric acid increased expression levels of tyrosinase and MITF proteins in melanocytes in a dose-dependent manner (Figure 1f). Levels of tyrosinase and MITF proteins increased by conditioned media from GDA-overexpressing keratinocytes along with uric acid were reduced when GDA-overexpressing keratinocytes were treated with allopurinol, a xanthine oxidase inhibitor (Figure 1g). 
a
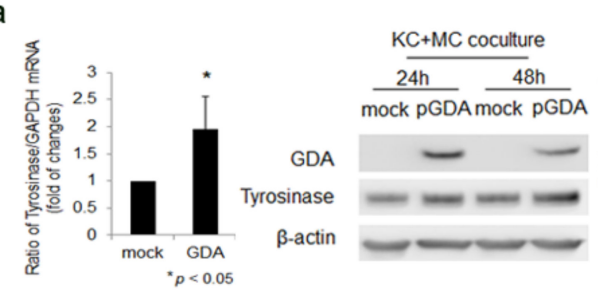

b

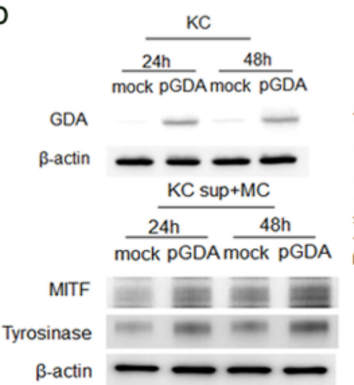

d
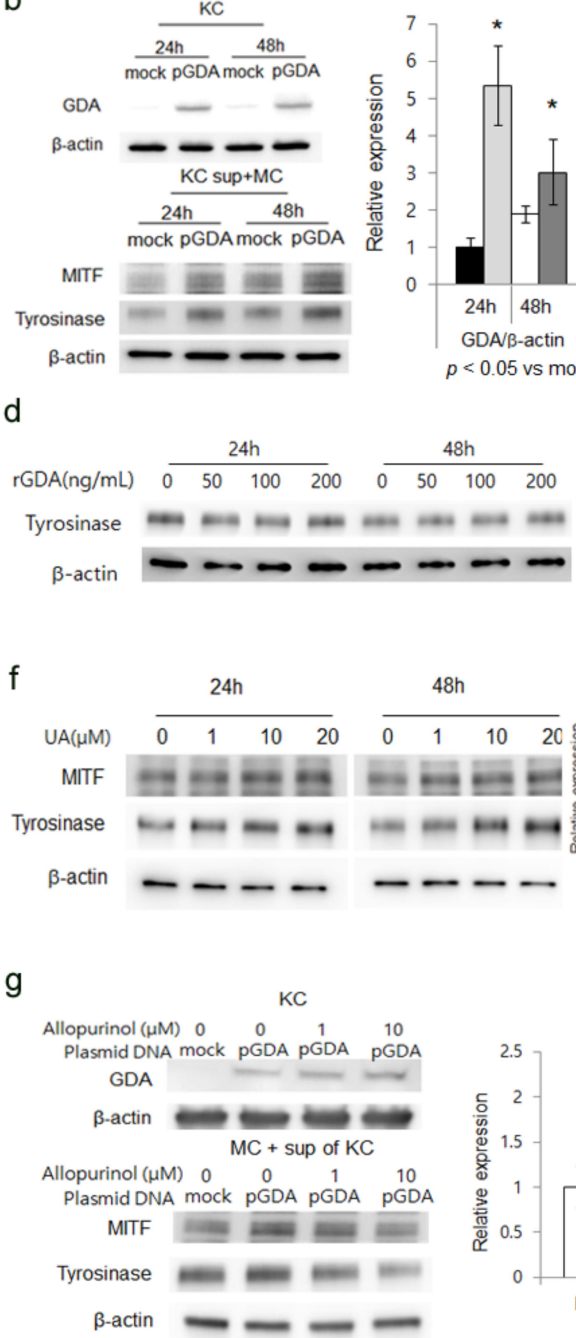

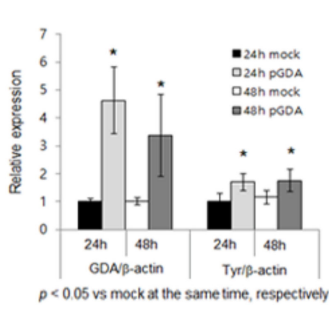

c
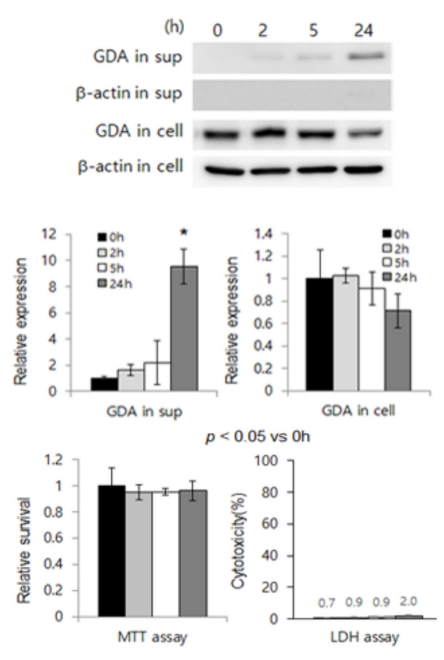

e

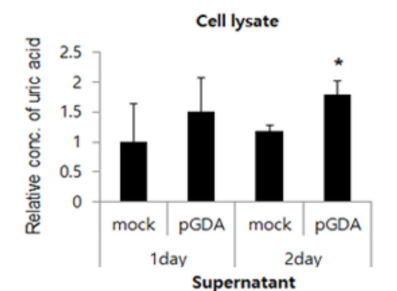

24h Tyrip.action 48 h
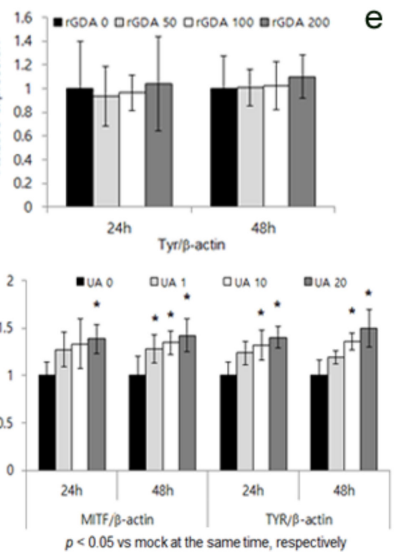

Figure 1. GDA upregulation in keratinocytes stimulates melanogenesis by releasing uric acid. (a) Real-time PCR and Western bot analyses for relative ratios of tyrosinase mRNA and protein levels, respectively, in primary cultured normal human skin melanocytes (MC) cocultured with GDA-overexpressing keratinocytes (KC). (b) Western blot analysis to determine relative ratios of tyrosinase and MITF protein levels in normal melanocytes treated with supernatants from GDAoverexpressing keratinocytes. (c) Western blot analysis for relative ratios of GDA protein levels in culture supernatants and GDA-overexpressing keratinocytes, whose viability and cytotoxicity were measured by MTT and LDH assays, respectively. (d) Western blot analysis for relative ratios of tyrosinase in normal melanocytes treated with recombinant GDA (rGDA). (e) Uric acid assay for measuring concentrations of uric acid in cell lysates and culture supernatants from keratinocytes after GDA overexpression for 2 days. (f) Western blot analysis for relative ratios of tyrosinase and MITF proteins in normal melanocytes treated with various concentrations of uric acid (UA). (g) Western blot analysis for relative ratios of tyrosinase and MITF proteins in normal melanocytes treated with supernatants from GDA-overexpressing keratinocytes cultured in the presence of various concentrations of allopurinol along with uric acid concentrations. $\beta$-Actin and GAPDH were used as internal controls for Western blot analysis and real-time PCR, respectively. Data represent means \pm SD of four independent experiments. 


\subsection{Uric Acid Is Secreted from Keratinocytes through ABCG2}

ABCG2 is one of main urate transporters for uric acid excretion expressed on apical membranes in several tissues [17], including interfollicular keratinocyte progenitor cells [18]. In the present study, expression levels of ABCG2 in epidermal keratinocytes and role of ABCG2 in the excretion of uric acid from epidermal keratinocytes were examined. Levels of ABCG2 protein, which was detectable in keratinocytes, were increased by GDA overexpression (Figure 2a). Treatment with Ko143 [(3S,6S,12aS)-1,2,3,4,6,7,12,12a-octahydro9-methoxy-6-(2-methylpropyl)-1,4-dioxopyrazino[ $\left.1^{\prime}, 2^{\prime}: 1,6\right]$ pyrido[3,4-b]indole-3-propanoic acid 1,1-dimethylethyl ester], an inhibitor of ABCG2 [19], reduced uric acid concentrations induced by GDA overexpression in keratinocyte culture supernatants, but increased them in keratinocyte lysates (Figure $2 b$ ). ABCG2 knockdown decreased tyrosinase protein levels in normal melanocytes cocultured with GDA-overexpressing keratinocytes (Figure 2c). $A B C G 2$ knockdown reduced uric acid concentrations in culture supernatants, but increased them in cell lysates from GDA-overexpressing keratinocytes (Figure 2d). Immunofluorescence study for skin specimens of patients with seborrheic keratosis with stronger GDA staining intensities using anti-ABCG2 and anti-uric acid antibodies showed stronger staining intensities in lesional epidermis (Figure 2e).

a
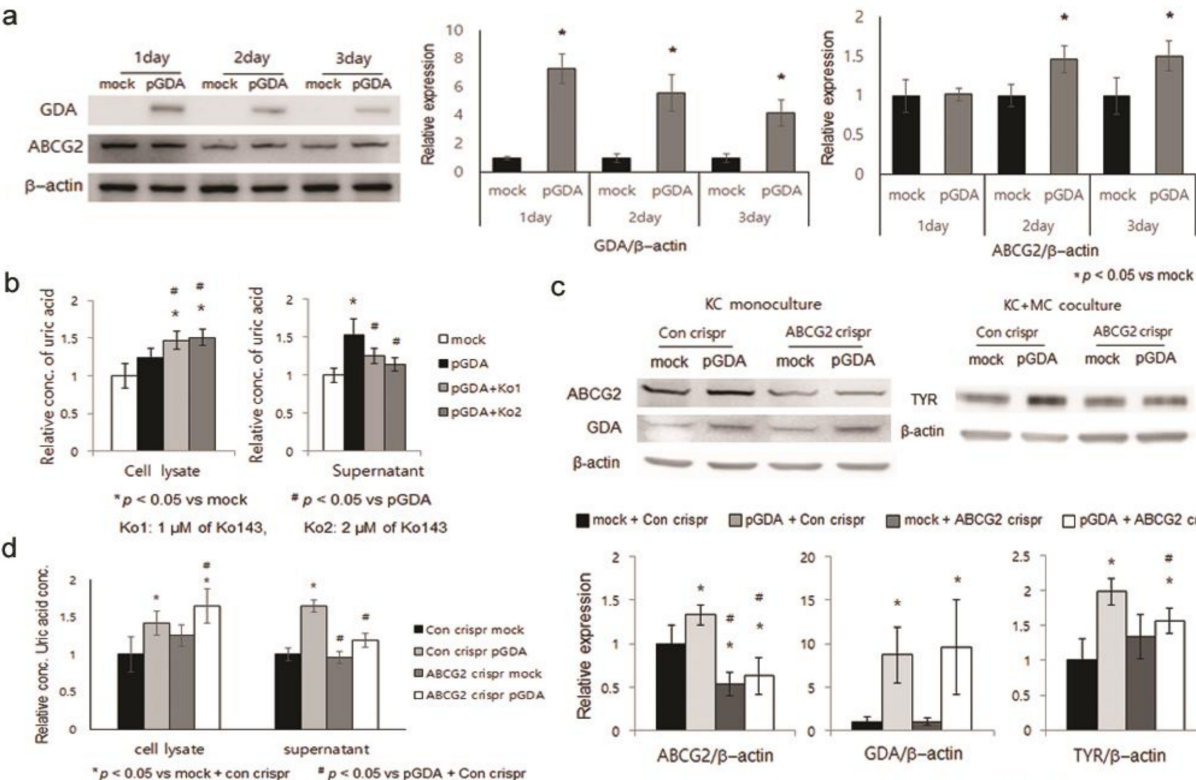
$p<0.05$ vs mock
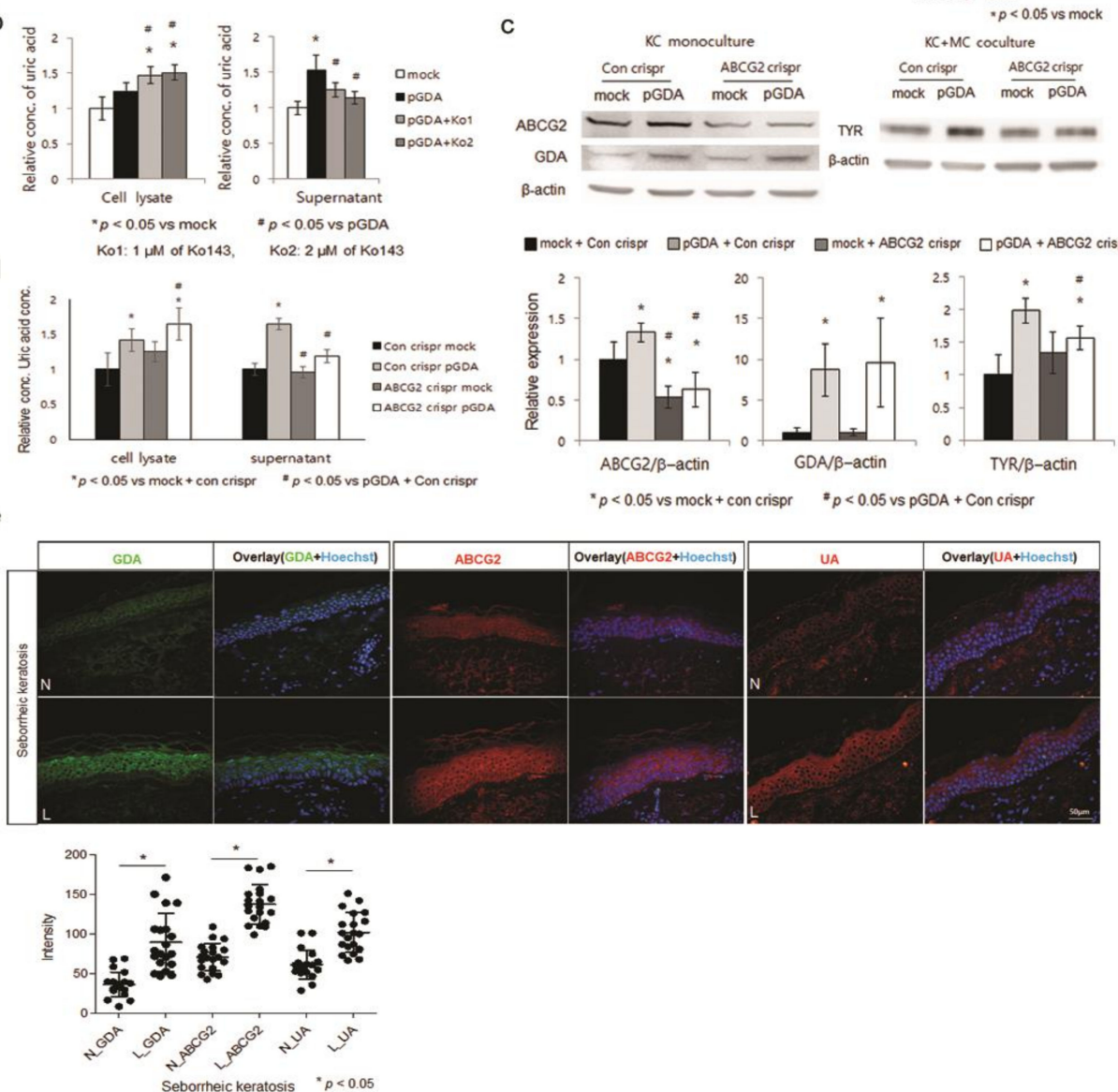

Figure 2. Uric acid is secreted from keratinocytes through ABCG2. (a) Western blot analysis for relative ratios of ABCG2 protein levels in keratinocytes after GDA overexpression. (b) Uric acid assay determining concentrations of uric acid in cell 
lysates and culture supernatants from GDA-overexpressing keratinocytes in the presence of Ko143 at different concentrations up to $24 \mathrm{~h}$. (c) Western blot analysis for relative ratios of tyrosinase protein levels in melanocytes (MC) applied with supernatants of GDA-overexpressing keratinocytes (KC) with or without $A B C G 2$ knockdown. (d) Uric acid assay for concentrations of uric acid in cell lysates and culture supernatants from GDA-overexpressing keratinocytes with or without ABCG2 knockdown. (e) Representative immunofluorescent staining using anti-GDA and anti-ABCG2 antibodies in lesional (L) skin samples compared to that in non-lesional $(\mathrm{N})$ skin samples from four patients with seborrheic keratosis. Nuclei were counter-stained with Hoechst 33258 (Bar $=0.05 \mathrm{~mm}$ ). Staining intensities were measured for five randomly selected areas in each image using a Wright Cell Imaging Facility ImageJ software. $\beta$-Actin was used as an internal control for Western blot analysis. Data represent means \pm SD of four independent experiments.

\subsection{ABCG2 Action Is Facilitated by PDZK1 in Keratinocytes}

PDZK1 plays a pivotal role in the regulation of ABCG2 in human intestinal cell lines [20]. Therefore, the role of GDA in PDZK1 expression and the role of PDZK1 in ABCG2 regulation were examined in epidermal keratinocytes. PDZK1 protein levels in keratinocytes were increased by GDA overexpression (Figure 3a). PDZK1 overexpression increased levels of ABCG2 (Figure $3 \mathrm{~b}$ ). Immunoprecipitation assay showed a detectable binding between PDZK1 and ABCG2 in PDZK1-overexpressing keratinocytes (Figure 3c). Staining intensity with an anti-ABCG2 antibody was stronger in lesional epidermis compared to normal epidermis of seborrheic keratosis showing increased PDZK1 staining intensity (Figure 3d).

a
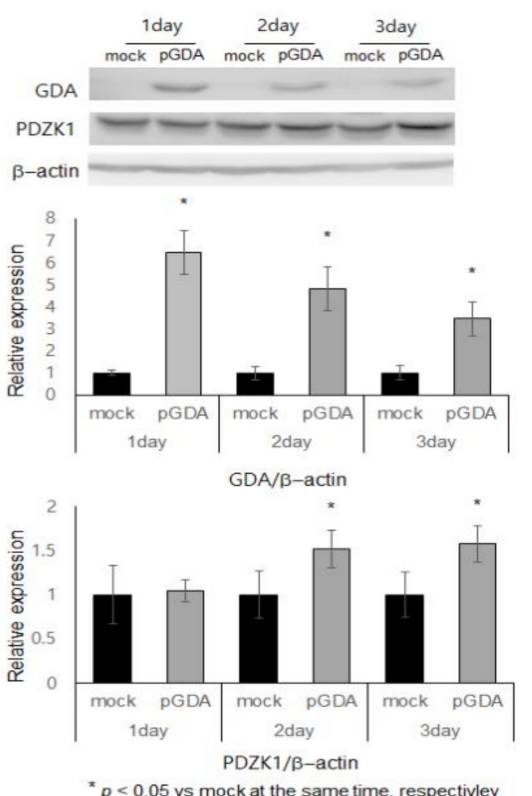

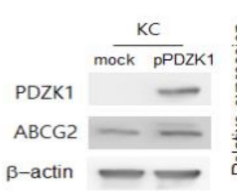

C
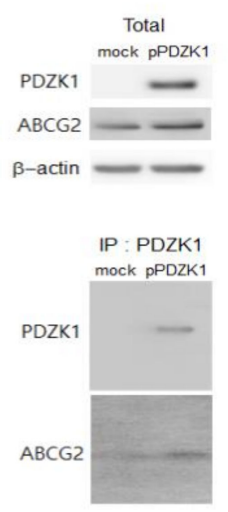
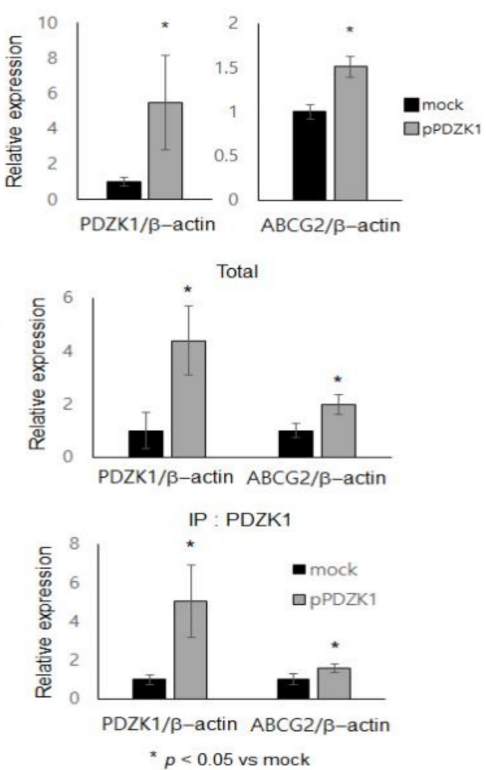

d

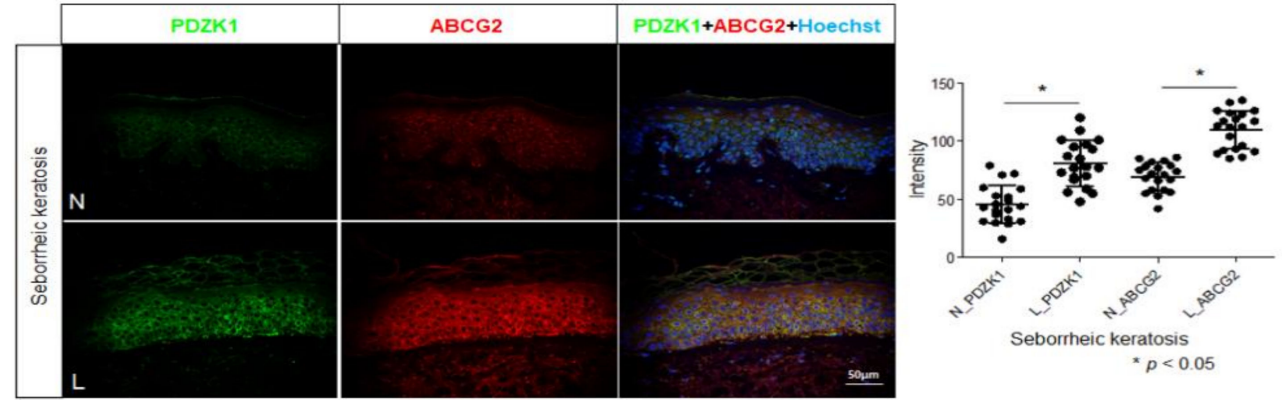

Figure 3. ABCG2 action is facilitated by PDZK1 in keratinocytes. (a) Western blot analysis for relative ratios of PDZK1 protein levels in keratinocytes after GDA overexpression. (b) Western blot analysis for relative ratios of ABCG2 protein 
levels in keratinocytes (KC) with PDZK1 overexpression. (c) Immunoprecipitation using anti-PDZK1 and anti-ABCG2 antibodies in cultured keratinocytes with or without PDZK1 overexpression. (d) Representative immunofluorescent staining using anti-PDZK1 and anti-ABCG2 antibodies in lesional (L) skin samples compared to that in non-lesional (N) skin samples from four patients with seborrheic keratosis. Nuclei were counter-stained with Hoechst 33258 (Bar $=0.05 \mathrm{~mm})$. Staining intensities were measured for five randomly selected areas in each image using a Wright Cell Imaging Facility ImageJ software. $\beta$-Actin was used as an internal control for Western blot analysis. Data represent means \pm SD of four independent experiments.

2.4. Uric Acid Uptake through URAT1 in Melanocytes, Stimulating Melanogenesis via p38 MAPK

Uric acid was excreted from keratinocytes through ABCG2 (Figures 3a and 4d) with the help of PDZK1 (Figure $3 \mathrm{a}-\mathrm{c}$ ). Uric acid should be taken by melanocytes to induce melanogenesis. URAT1 is a major transporter responsible for the reabsorption of urate in kidney and from blood $[21,22]$. Therefore, the presence and the role of URAT1 in uric acid uptake were examined in melanocytes. Treatment with uric acid increased levels of URAT1 in primary cultured epidermal melanocytes (Figure 4a). Levels of tyrosinase and MITF proteins were also increased by uric acid (Figure $4 \mathrm{a}$ ). However, their levels were reduced by probenecid, an inhibitor of URAT1 [23], along with intracellular uric acid concentrations (Figure 4b). URAT1 knockdown also reduced levels of intracellular uric acid along with tyrosinase and MITF proteins in melanocytes treated with exogenous uric acid (Figure 4c). These increase of tyrosinase and MITF proteins after the application of supernatants from GDA overexpressing keratinocytes were also reduced by URAT1 knockdown (Figure 4d). Immunofluorescence study for skin specimens of seborrheic keratosis using anti-URAT1 and anti-cKIT, a receptor tyrosine kinase expressed in melanocytes [24,25], antibodies showed stronger staining intensities in lesional melanocytes (Figure 4e). Uric acid activated p38 mitogen-activated protein kinase (MAPK), but not ERK or JNK, in melanocytes in a dose-dependent manner (Figure 4f). SB203580, a p38 MAPK inhibitor, reduced levels of tyrosinase and MITF proteins in the presence of uric acid (Figure $4 \mathrm{~g}$ ). 
a
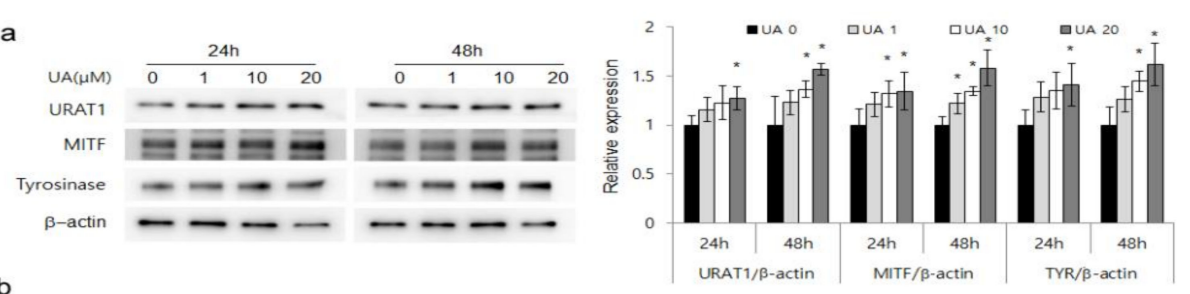

b
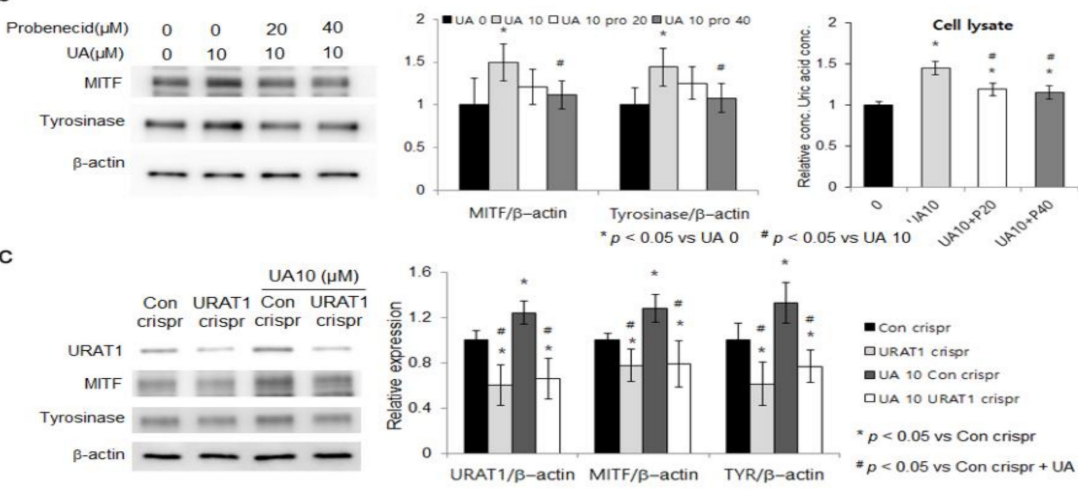

- Con crispr

qua 10 con crisper

QUA 10 URAT1 1 crispr

* $p<0.05$ vs Con crispr

$p<0.05$ vs Con crispr + UA 10

d

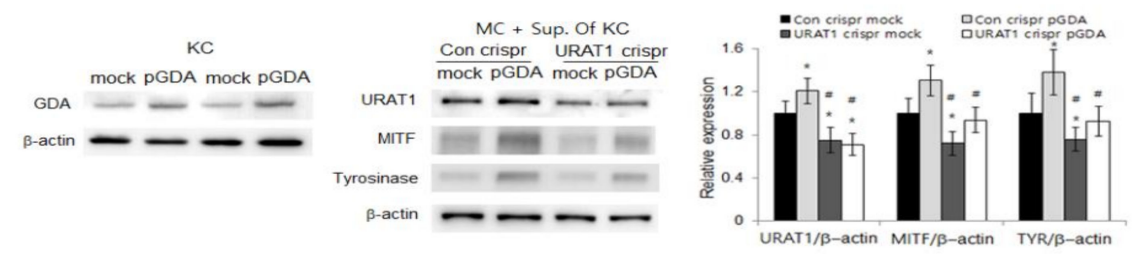

e
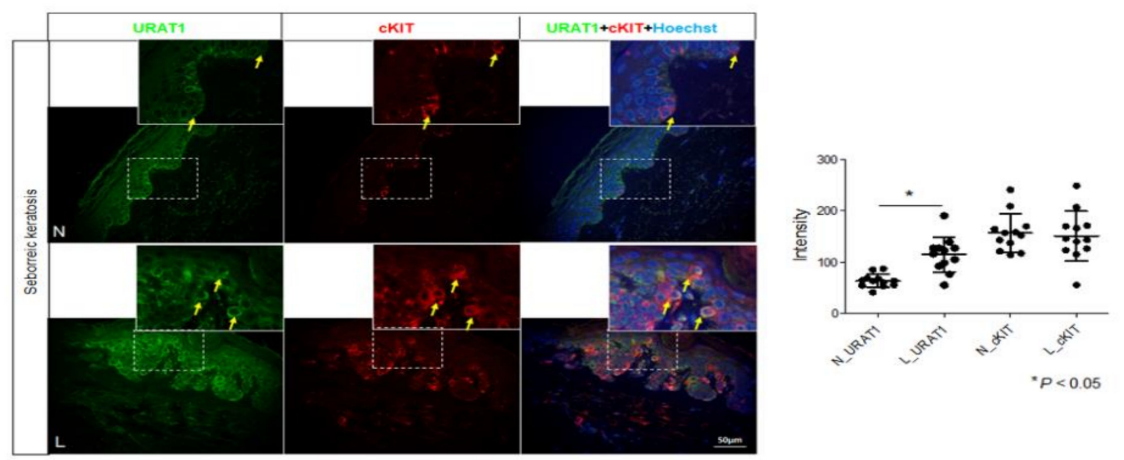

f

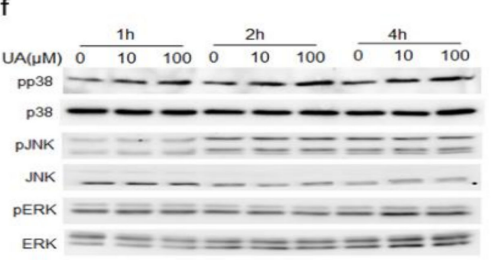

g

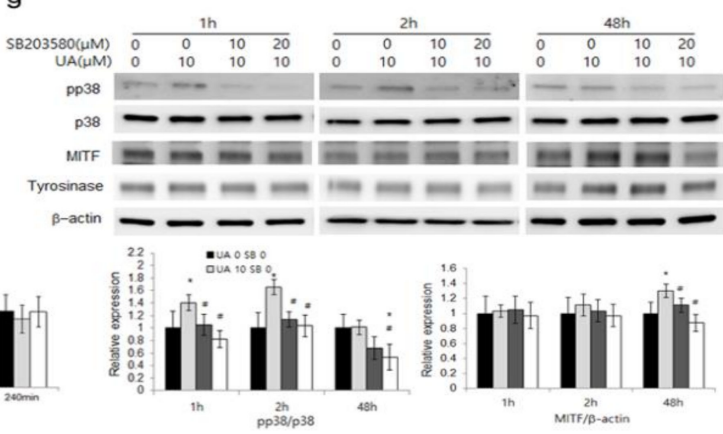

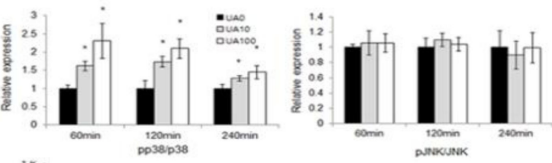

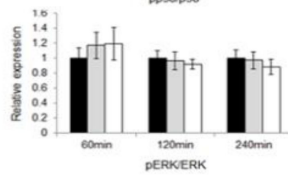

II In It

$" p<0.05$ vs UA $O$

$-p<0.05$ vs UA 10 SBO

Figure 4. Uric acid uptake through URAT1 in melanocytes, stimulating melanogenesis via p38 MAPK. (a) Western blot analysis for relative ratios of URAT1, tyrosinase, and MITF proteins in normal melanocytes treated with different 
concentrations of uric acid (UA). (b-c) Relative ratios of tyrosinase and MITF proteins and intracellular uric acid concentrations in normal melanocytes treated with different concentrations of probenecid (b) and in control or URAT1-knockdown melanocytes (c) in the absence or presence of exogenous uric acid based on Western blot analysis. (d) Relative ratios of tyrosinase and MITF proteins in control or URAT1-knockdown melanocytes (MC) treated with supernatants from GDAoverexpressing keratinocytes (KC) by Western blot analysis. (e) Representative immunofluorescent staining using anti-cKIT and anti-URAT1 antibodies in lesional (L) skin samples compared to that in non-lesional (N) skin samples from three patients with seborrheic keratosis. Nuclei were counter-stained with Hoechst 33258 (Bar $=0.05 \mathrm{~mm})$. Staining intensities were measured for four randomly selected areas in each image using a Wright Cell Imaging Facility ImageJ software. $(\mathbf{f}, \mathbf{g})$ Western blot analysis for relative ratios of p38 MAPK, JNK, and ERK proteins in normal melanocytes treated with different concentrations of uric acid in the absence (f) or presence of SB203580 (g). $\beta$-Actin was used as an internal control for Western blot analysis. Data represent means \pm SD of four independent experiments.

\section{Discussion}

This study first examined whether GDA upregulation could be involved in skin hyperpigmentation. Increased levels of tyrosinase and MITF by coculturing with GDAoverexpressing keratinocytes (Figure 1a) suggested a role of GDA in enhancing melanogenesis. In fact, GDA upregulation has been reported in melasma [26-28], a representative pigmentary skin disorder, in addition to seborrheic keratosis [5]. We have also detected upregulation of GDA in melasma (data not shown). Hyperpigmentation is a common clinical finding of seborrheic keratosis and melasma. In addition, UV radiation is one of main causative factors of melasma [29-33]. Skin aging is also emerging as a cause of melasma [31,32,34,35], indicating that seborrheic keratosis shares certain causes with melasma. The identified role of GDA upregulation in UV-induced keratinocyte senescence [5] suggested that upregulated GDA contributed to the hyperpigmention, particularly related to photoaging.

Based on the result that GDA upregulation stimulated melanogenesis, its mechanism was then investigated. Melanogenesis in melanocytes was also enhanced by conditioned media obtained from GDA-overexpressing keratinocytes (Figure 1b), suggesting the presence of $G D A$ or other factors for stimulating melanogenesis in conditioned media. In fact, GDA level was increased in the conditioned media in the absence of keratinocyte damage (Figure 1c). However, receptor of GDA has not been identified in melanocytes yet. There was no enhancement of melanogenesis from melanocytes by recombinant GDA either (Figure 1d), suggesting roles of melanogenic factors other than GDA released from $G D A$-overexpressing keratinocytes in melanogenesis. An interaction between melanogenic growth factors and GDA in keratinocytes has been mentioned [28]. We also found that levels of melanogenic growth factors such as basic fibroblast growth factor and SCF were increased in keratinocytes by GDA over expression (Supplementary Figure S1). Although the exact mechanism involved in the upregulation of melanogenic growth factors by GDAoverexpressing keratinocytes was not clarified, this study was focused on the role of GDA in melanogenesis related to photoaging through its metabolic end product based on the role of uric acid in UV-induced keratinocyte senescence [5]. Increased uric acid levels in culture supernatants of $G D A$-overexpressing keratinocytes which stimulated melanogenesis (Figure 1e) could support the need for investigating the role of GDA via uric acid. Levels of MITF and tyrosinase enhanced by exogenous uric acid (Figure 1f). Increased uric acid levels along with MITF and tyrosinase levels in GDA-overexpressing keratinocytes were restored by allopurinol (Figure 1g). These findings indicate a significant role of uric acid in GDA-induced melanogenesis.

Although keratinocytes generated uric acid (Figure 1e), how uric acid was released from keratinocytes was unclear. Therefore, the role of a transporter for uric acid excretion, ABCG2 [17], was examined. ABCG2 levels were upregulated in GDA-overexpressing keratinocytes (Figure 2a). Ko143, an ABCG2 inhibitor, and ABCG2 knockdown reduced GDA-induced uric acid concentrations in supernatants but increased them in cell lysates (Figure $2 b-d$ ). These results indicated the necessity of ABCG2 upregulation for excretion of uric acid in GDA-overexpressing keratinocytes. In addition, GDA-induced tyrosinase levels 
were reduced by ABCG2 knockdown (Figure 2c), indicating that ABCG2 was involved in melanogenesis by regulating uric acid excretion. Stronger staining intensities with anti-ABCG2 and anti-uric acid antibodies in lesional skin of seborrheic keratosis with GDA upregulation (Figure 2e) supported an association between ABCG2 and uric acid in seborrheic keratosis. Although the regulatory role of PDZK1 in ABCG2 [20] has not been identified in the skin, PDZK1 was upregulated in keratinocytes by GDA overexpression (Figure 3a). In addition, ABCG2 levels were enhanced by PDZK1 overexpression (Figure 3b) and the binding between ABCG2 and PDZK1 (Figure 3c), indicating that the role of ABCG2 in GDA-induced melanogenesis could be facilitated by PDZK1. PDZK1 might play a role as a scaffold as shown in estrogen-related skin pigmentation [36]. Stronger staining intensities with anti-PDZK1 and anti-ABCG2 antibodies in lesional epidermis of seborrheic keratosis (Figure 3d) also supported clinical implications of their relations.

In order to participate in melanogenesis, uric acid excreted from keratinocytes should be taken by melanocytes. It has been reported that URAT1 is a transporter for uric acid resorption [21,22]. Although such data are unavailable for the skin, levels of URAT1 were enhanced by uric acid in a dose-dependent manner (Figure 4a) while levels of tyrosinase, MITF as well as intracellular uric acid were reduced by a URAT1 inhibitor (Figure $4 b$ ) or URAT1 knockdown (Figure 4c,d), indicating an important role of URAT1 in uric acidstimulated melanogenesis by uric acid reabsorption in melanocytes. Through continuing efforts to find URAT1 inhibitors to control hyperuricemia more safely, some natural products such as nobiletin (polymethoxyflavonoid), hesperetin, naringenin (flavanones), and dioscin (steroidal saponin) have been identified as materials suitable for hyperuricemia control [37,38]. Although a few studies have examined effects of these natural products on melanogenesis inhibition, result from some of these URAT1 inhibitors [39-43] supported a role of URAT1 in melanogenesis. Stronger staining intensity with an anti-URAT1 antibody in lesional melanocytes, which were identified by positive staining with anti-cKIT antibody, from patients with seborrheic keratosis (Figure 4e) reinforced a role of URAT1 in hyperpigmentation of seborrheic keratosis.

Finally, how the uric acid taken by melanocytes stimulated melanogenesis was examined. MAPK is one of melanogenesis-related signaling pathways [44-46]. Activation of p38 MAPK is presented in the signaling pathway for uric acid action [47-49]. Examining the role of MAPK in uric acid induced melanogenesis showed an activation of p38 MAPK by uric acid (Figure $4 \mathrm{f}$ ) and a reduction of uric acid-induced melanogenesis by a 38 MAPK inhibitor (Figure 4g). These results indicate that uric acid could stimulate melanogenesis through p38 MAPK signaling pathway.

In summary, GDA upregulation in keratinocytes can stimulate melanogenesis via uric acid generation. Uric acid is then excreted by the upregulation and interaction between ABCG2 and PDZK1. Excreted uric acid is then reabsorbed by melanocytes via URAT1 upregulation, leading to melanogenesis through p38 MAPK activation (Figure 5). Considering that GDA is involved in UV-induced keratinocyte senescence, these events support the association of hyperpigmentation with photoaging in seborrheic keratosis. 


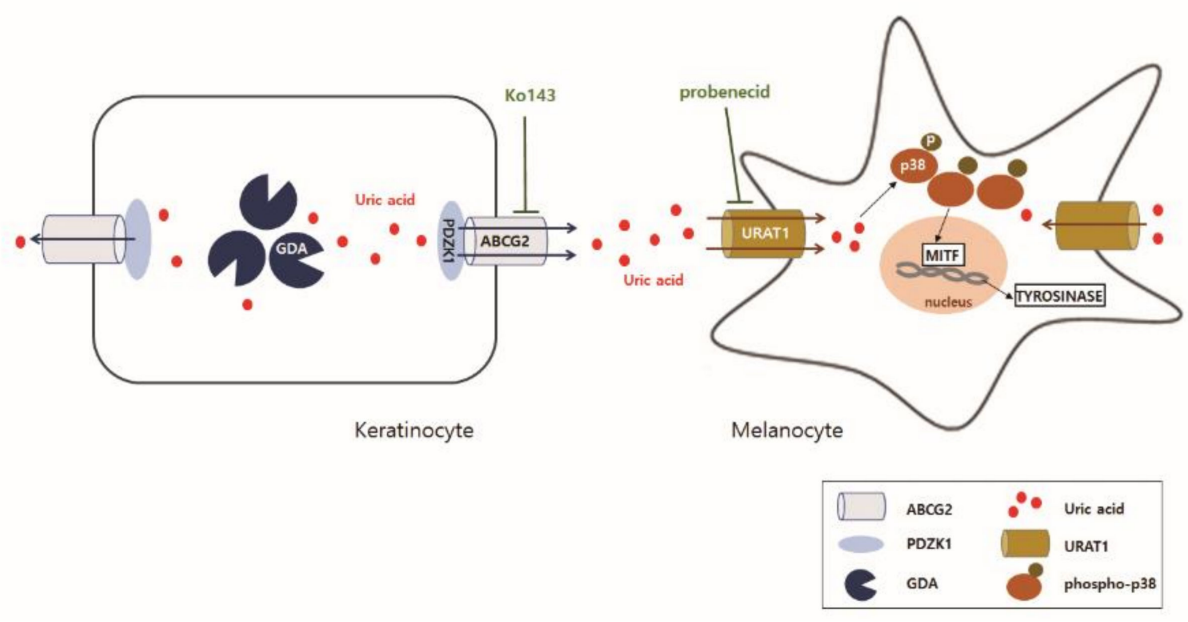

Figure 5. Schematic view of GDA-induced melanogenesis via uric acid.

Excretion of uric acid is induced by the upregulation and interaction between ABCG2 and PDZK1 in GDA-overexpressing keratinocytes. Excreted uric acid is reabsorbed by melanocytes via URAT1 upregulation through p38 MAPK activation, leading to melanogenesis.

\section{Materials and Methods}

\subsection{Patients}

Four patients diagnosed with seborrheic keratosis with a mean age of 64.5 were included in the study. This study was approved by the Institutional Review Board of Dongguk University Ilsan Hospital (the approval No. 2012-01-032). It was conducted according to the Declaration of Helsinki Principles. After obtaining informed written consent from each patient, pairs of hyperpigmented and adjacent normally pigmented skin specimens taken for biopsy were used for direct comparisons through real-time PCR and immunohistochemistry.

\subsection{Normal Human Epidermal Cell Culture}

Adult skin specimens obtained from Caesarean sections and circumcisions were used to establish cell culture for keratinocytes and melanocytes [5,50,51]. Keratinocyte cultures were obtained by suspending individual epidermal cells derived according to published methods, in EpiLife Medium supplemented with bovine pituitary extract (BPE), bovine insulin (BI), hydrocortisone, human epidermal growth factor, and bovine transferrin (BT) (Thermo Fisher Scientific, Waltham, MA, USA). Melanocyte cultures were obtained by suspending individual epidermal cells in Medium 254 supplemented with BPE, fetal bovine serum (FBS), BI, hydrocortisone, bFGF, BT, heparin, and phorbol 12-myristate 13-acetate (Thermo Fisher Scientific, Waltham, MA, USA). Keratinocytes between passages 3 and 5 and melanocytes at passages between 8 and 25 were used for real-time PCR, western blot, immunoprecipitation, 3-(4,5-dimethylthiazol-2yl)-2,5-diphenyltetrazolium bromide (MTT; Dongin Biotech. Seoul, Korea) assay, and lactate dehydrogenase (LDH; Dongin Biotech) assay with or without gene transfection.

\subsection{Overexpression of GDA and Knockdown of ABCG2, or URAT1}

Keratinocytes or melanocytes were transfected with $25 \mathrm{nM}$ CRISPR-CAS9 sgRNA for human ABCG2, URAT1, or a negative control sgRNA (Integrated DNA Technologies, San Diego, CA, USA) using a CRISPRMAX transfection reagent (Thermo Fisher Scientific, Waltham, MA, USA). pCMV vector containing GDA gene [5] was transfected into cells using Lipofectamine 2000 (Thermo Fisher Scientific). Cells were used for experiments at appropriate time post-transfection. 


\subsection{Cell Viability and Cytotoxicity Test}

To measure cell viability, cells were incubated with MTT for $4 \mathrm{~h}$. Precipitated formazan was dissolved in dimethyl sulfoxide (DMSO). The optical density was measured at $570 \mathrm{~nm}$ with background subtraction at $630 \mathrm{~nm}$ using a spectrophotometer. LDH assay performed following the manufacturer's instruction for measuring cell cytotoxicity.

\subsection{Recombinant GDA Treatment}

Melanocytes $\left(2 \times 105\right.$ cells $/ 35 \mathrm{~mm}$ diameter well with an area of $\left.\sim 9.6 \mathrm{~cm}^{2}\right)$ were treated with various concentrations of recombinant GDA (50, 100, and $200 \mathrm{ng} / \mathrm{mL}$ ) (BioVision, Milpitas, CA, USA) up to 3 days.

\subsection{Uric Acid Assay}

Concentrations of uric acid were determined fluorometically $(\mathrm{Ex} / \mathrm{Em}=535 / 587 \mathrm{~nm})$ in cell lysates or culture supernatants using a uric acid assay kit (Abcam, Cambridge, UK), following the manufacturer's instructions.

\subsection{Treatment with Allopurinol or Ko143}

Keratinocytes $\left(2 \times 105\right.$ cells $\left./ 9.6 \mathrm{~cm}^{2}\right)$ were transfected with GDA. Four hours later, cells were treated with different concentrations of allopurinol $(1$ and $10 \mu \mathrm{M})$ or Ko143 (1 and $2 \mu \mathrm{M}$ ) (Sigma-Aldrich, St. Louis, MO, USA) for 2 days or $24 \mathrm{~h}$, respectively. Cells or culture supernatants were harvested for melanocyte culture or uric acid assay.

\subsection{Uric Acid Treatment with/without Probenecid}

Melanocytes $\left(2 \times 105\right.$ cells $\left./ 9.6 \mathrm{~cm}^{2}\right)$ were treated with appropriate concentrations of uric acid (Sigma-Aldrich) with or without probenecid (20 and $40 \mu \mathrm{M})$ (Sigma-Aldrich) for $24 \mathrm{~h}$. Cells or culture supernatants were harvested for uric acid assay.

\subsection{Real-Time PCR}

cDNA was synthesized from the total RNA using the cDNA Synthesis Kit for RT-PCR (Promega, Fichburg, WI, USA). The amount of target mRNA was quantified by real-time PCR using a Light Cycler real-time PCR machine (Roche, Penzberg, Germany). The relative amount of mRNAs was calculated as the ratio of each target relative to glyceraldehyde 3-phosphate dehydrogenase (GAPDH). Primer sequences used were as follows: GDA, 5' catagtgacaccacgtttttcc- $3^{\prime}$ (Forward) and $5^{\prime}$-cgattttcacttatatggctctga- $3^{\prime}$ (Reverse); Tyrosinase, $5^{\prime}$-agcatcattcttctcctcttgg- $3^{\prime}$ (Forward) and $5^{\prime}$-gcataaagactgatggctgttg-3' (Reverse); GAPDH, $5^{\prime}$-tccactggcgtcttcacc-3' (Forward) and 5'-ggcagagatgatgacccttt- $3^{\prime}$ (Reverse).

\subsection{Western Blot Analysis}

Equal amounts of extracted proteins were resolved and transferred to nitrocellulose membranes. Each membrane was incubated with one of the following antibodies against ABCG2, GDA, PDZK1, tyrosinase, and URAT1 (mouse monoclonal; Santa Cruz Biotechnology, Dallas, TX, USA), MITF, phospho-ERK, ERK, phospho-p38, p38, and JNK (rabbit polyclonal; Cell Signaling Technology, Beverly, MA, USA), phospho-JNK (mouse monoclonal; cell signaling technology) and $\beta$-Actin (mouse monoclonal; Sigma-Aldrich). After incubating with appropriate anti-mouse or anti-rabbit horseradish peroxidase-conjugated antibodies (Thermo Fisher Scientific), an enhanced chemiluminescence solution (Thermo Fisher Scientific) was applied and signals were captured with an image reader (LAS-3000; Fuji Photo Film, Tokyo, Japan). The protein bands were then analyzed by densitometry.

\subsection{Immunoprecipitation}

Supernatants of cell lysates were incubated with an anti-PDZK1 antibody and resin of Pierce ${ }^{\mathrm{TM}}$ Direct IP kit (Thermo Fisher Scientific) at $4{ }^{\circ} \mathrm{C}$. Eluted resin-bound proteins were analyzed by immunoblotting with anti-ABCG2 or anti-ABCG2 antibody. 


\subsection{Immunohistochemistry}

After deparaffinization and rehydration, sections were preincubated with $3 \%$ bovine serum albumin. These sections were reacted sequentially with the an anti-GDA, antiPDZK1, or anti-URAT1 antibody and 1:200 Alexa Fluor-labeled goat anti-mouse IgG (488; Molecular Probes, Eugene, OR, USA), with anti-ABCG2 and Alexa Fluor-labeled goat anti-mouse IgG (594; Molecular Probes), or with anti-cKit (Cell signaling technology), anti-uric acid (Santa Cruz Biotechnology) antibody, and Alexa Fluor-labeled goat antirabbit IgG (594; Molecular Probes). Nuclei were counterstained with Hoechst 33258 (Sigma-Aldrich). Fluorescence images were evaluated using an image analysis system (Dp Manager 2.1; Olympus Optical Co., Tokyo, Japan) and a Wright Cell Imaging Facility (WCIF) ImageJ software 1.53e (http:/ / www.uhnresearch.ca/facilities/wcif/imagej accessed on 9 August 2021).

\subsection{Statistical Analysis}

All statistical analyses were performed using GraphPad Prism 5.03 (GraphPad Software, La Jolla, CA, USA). A $p$-value of $<0.05$ was considered significant. Statistical comparisons between two groups were performed using two-tailed Student's unpaired $t$-test (parametrical data). For comparisons among multiple groups, one-way Analysis of Variance (ANOVA) was used. For human sample data, differences between non-lesions and lesions were assessed with the Mann-Whitney U test. Values of mean \pm SD were calculated for in vitro experimental data.

\section{Conclusions}

GDA upregulation stimulated melanogenesis. Regarding the mechanism of GDAinduced hyperpigmentation, GDA upregulation enhanced melanogenesis via uric acid generation. More uric acid was excreted by GDA-overexpressing keratinocytes through ABCG2 with the help of PDZK1. Excreted uric acid, which was reabsorbed by melanocytes via URAT1 upregulation, enhanced melanogenesis through p38 MAPK activation. Although other factors could be involved in GDA-induced hyperpigmentation, generation of uric acid, a metabolic end product, could contribute to GDA-induced-melanogenesis.

\section{Patents}

It was described in the Materials and Methods of the original.

Supplementary Materials: The following are available online at https://www.mdpi.com/xxx/s1.

Author Contributions: Conceptualization: A.-Y.L. and K.A.C.; Data Curation: A.-Y.L. and K.A.C.; Formal Analysis: A.-Y.L. and K.A.C.; Funding Acquisition: A.-Y.L.; Investigation: A.-Y.L., K.A.C., I.S.K., and H.W.K.; Methodology: K.A.C.; Supervision: A.-Y.L., K.A.C., I.S.K., and H.W.K.; Validation: A.-Y.L. and K.A.C. Visualization: A.-Y.L. and K.A.C.; Writing Original Draft Preparation: A.-Y.L. and K.A.C. All authors have read and agreed to the published version of the manuscript.

Funding: National Research Foundation of Korea: NRF 2018R1A5A2023127. Korea Health Industry Development Institute: HP20C0131.

Institutional Review Board Statement: This study was approved by the Institutional Review Board of Dongguk University Ilsan Hospital (the approval No. 2012-01-032). It was conducted according to the Declaration of Helsinki Principles.

Informed Consent Statement: Scanned < Informed consent forms $>$ from four patients are attached with an English version of $<$ Certificate of Approval $>$.

Data Availability Statement: Not applicable.

Acknowledgments: This work was supported by the National Research Foundation of Korea (NRF) grant funded by the Korea government (MSIT) (No. NRF 2018R1A5A2023127). This research was also supported by a grant of the Korea Health Technology R\&D Project through the Korea Health Industry Development Institute (KHIDI), funded by the Ministry of Health \& Welfare, Republic of Korea (grant number: HP20C0131). 
Conflicts of Interest: The authors state no conflict of interest.

$\begin{array}{ll}\text { Abbreviations } \\ \text { GDA } & \begin{array}{l}\text { guanine deaminase } \\ \text { UV }\end{array} \\ \text { ultraviolet } \\ \text { SCF } & \text { stem cell factor } \\ \text { ABCG2 } & \text { ATP-binding cassette transporter, sub-family G, member } 2 \\ \text { URAT1 } & \text { urate transporter 1 } \\ \text { PDZK1 } & \text { PDZ domain containing 1 } \\ \text { MAPK } & \text { mitogen-activated protein kinase } \\ \text { Ko143 } & {[3 S, 6 S, 12 a S]-1,2,3,4,6,7,12,12 \text { a-octahydro-9-methoxy-6-[2-methylpropyl]-1,4-dioxopy- }} \\ & \text { razino }\left[1^{\prime}, 2^{\prime}: 1,6\right] \text { pyrido[3,4-b]indole-3-propanoic acid 1,1-dimethylethyl ester] }\end{array}$

\section{References}

1. Hahnel, E.; Blume-Peytavi, U.; Trojahn, C.; Dobos, G.; Jahnke, I.; Kanti, V.; Richter, C.; Lichterfeld-Kottner, A.; Bartels, N.G.; Kottner, J. Prevalence and associated factors of skin diseases in aged nursing home residents: A multicentre prevalence study. BMJ Open 2017, 7, e018283. [CrossRef]

2. Kumar, D.; Das, A.; Bandyopadhyay, D.; Chowdhury, S.N.; Das, N.K.; Sharma, P.; Kumar, A. Dermatoses in the Elderly: Clinico-Demographic Profile of Patients Attending a Tertiary Care Centre. Indian J. Dermatol. 2021, 66, 74-80.

3. Wollina, U. Recent advances in managing and understanding seborrheic keratosis. F1000Research 2019, 8, F1000. [CrossRef]

4. Todorova, K.; Mandinova, A. Novel approaches for managing aged skin and nonmelanoma skin cancer. Adv. Drug. Deliv. Rev. 2020, 153, 18-27. [CrossRef]

5. Cheong, K.A.; Lee, A.Y. Guanine deaminase stimulates ultraviolet-induced keratinocyte senescence in seborrhoeic keratosis via guanine metabolites. Acta Derm. Venereol. 2020, 100, adv00109. [CrossRef] [PubMed]

6. Lee, A.Y.; Noh, M. The regulation of epidermal melanogenesis via cAMP and/or PKC signaling pathways: Insights for the development of hypopigmenting agents. Arch. Pharm. Res. 2013, 36, 792-801. [CrossRef] [PubMed]

7. Molinar, V.E.; Taylor, S.C.; Pandya, A.G. What's new in objective assessment and treatment of facial hyperpigmentation? Dermatol. Clin. 2014, 32, 123-135. [CrossRef]

8. Liu, Z.; Que, S.; Zhou, L.; Zheng, S. Dose-response Relationship of Serum Uric Acid with Metabolic Syndrome and Non-alcoholic Fatty Liver Disease Incidence: A Meta-analysis of Prospective Studies. Sci. Rep. 2015, 5, 14325. [CrossRef] [PubMed]

9. Yuan, H.; Yu, C.; Li, X.; Sun, L.; Zhu, X.; Zhao, C.; Zhang, Z.; Yang, Z. Serum Uric Acid Levels and Risk of Metabolic Syndrome: A Dose-Response Meta-Analysis of Prospective Studies. J. Clin. Endocrinol. Metab. 2015, 100, 4198-4207. [CrossRef]

10. King, C.; Lanaspa, M.A.; Jensen, T.; Tolan, D.R.; Sánchez-Lozada, L.G.; Johnson, R.J. Uric Acid as a Cause of the Metabolic Syndrome. Contrib. Nephrol. 2018, 192, 88-102.

11. Cortese, F.; Scicchitano, P.; Cortese, A.M.; Meliota, G.; Andriani, A.; Truncellito, L.; Calculli, G.; Giordano, P.; Ciccone, M.M. Uric Acid in Metabolic and Cerebrovascular Disorders: A Review. Curr. Vasc. Pharmacol. 2020, 18, 610-618. [CrossRef] [PubMed]

12. Goli, P.; Riahi, R.; Daniali, S.S.; Pourmirzaei, M.; Kelishadi, R. Association of serum uric acid concentration with components of pediatric metabolic syndrome: A systematic review and meta-analysis. J. Res. Med. Sci. 2020, 25, 43.

13. Cestari, T.F.; Dantas, L.P.; Boza, J.C. Acquired hyperpigmentations. An. Bras. Dermatol. 2014, 89, 11-25. [CrossRef]

14. Park, Y.J.; Kang, H.Y.; Lee, E.S.; Kim, Y.C. Differentiating confluent and reticulated papillomatosis from acanthosis nigricans. J. Cutan. Pathol. 2015, 42, 944-952. [CrossRef] [PubMed]

15. Huang, Y.; Chen, J.; Wang, X.; Li, Y.; Yang, S.; Qu, S. The Clinical Characteristics of Obese Patients with Acanthosis Nigricans and Its Independent Risk Factors. Exp. Clin. Endocrinol. Diabetes 2017, 125, 191-195.

16. Zhu, C.; Cui, R.; Gao, M.; Rampersad, S.; You, H.; Sheng, C.; Yang, P.; Sheng, H.; Cheng, X.; Bu, L.; et al. The Associations of Serum Uric Acid with Obesity-Related Acanthosis nigricans and Related Metabolic Indices. Int. J. Endocrinol. 2017, 2017, 5438157. [CrossRef]

17. Fujita, K.; Ichida, K. ABCG2 as a therapeutic target candidate for gout. Expert Opin. Ther. Targets 2018, 22, 123-129. [CrossRef]

18. Ma, D.; Chua, A.W.; Yang, E.; Teo, P.; Ting, Y.; Song, C.; Lane, E.B.; Lee, S.T. Breast cancer resistance protein identifies clonogenic keratinocytes in human interfollicular epidermis. Stem Cell Res. Ther. 2015, 6, 43. [CrossRef] [PubMed]

19. Stacy, A.E.; Jansson, P.J.; Richardson, D.R. Molecular pharmacology of ABCG2 and its role in chemoresistance. Mol. Pharmacol. 2013, 84, 655-669. [CrossRef]

20. Chen, M.; Lu, X.; Lu, C.; Shen, N.; Jiang, Y.; Chen, M.; Wu, H. Soluble uric acid increases PDZK1 and ABCG2 expression in human intestinal cell lines via the TLR4-NLRP3 inflammasome and PI3K/Akt signaling pathway. Arthritis Res. Ther. 2018, 20, 20. [CrossRef]

21. Anzai, N.; Miyazaki, H.; Noshiro, R.; Khamdang, S.; Chairoungdua, A.; Shin, H.-J.; Enomoto, A.; Sakamoto, S.; Hirata, T.; Tomita, K.; et al. The multivalent PDZ domain-containing protein PDZK1 regulates transport activity of renal urate-anion exchanger URAT1 via its C terminus. J. Biol. Chem. 2004, 279, 45942-45950. [CrossRef] 
22. Dong, Y.; Zhao, T.; Ai, W.; Zalloum, W.A.; Kang, D.; Wu, T.; Liu, X.; Zhan, P. Novel urate transporter 1 (URAT1) inhibitors: A review of recent patent literature (2016-2019). Expert Opin. Ther. Pat. 2019, 29, 871-879. [CrossRef]

23. Tan, P.K.; Ostertag, T.M.; Miner, J.N. Mechanism of high affinity inhibition of the human urate transporter URAT1. Sci. Rep. 2016, 6, 34995. [CrossRef] [PubMed]

24. Kitamura, R.; Tsukamoto, K.; Harada, K.; Shimizu, A.; Shimada, S.; Kobayashi, T.; Imokawa, G. Mechanisms underlying the dysfunction of melanocytes in vitiligo epidermis: Role of SCF/KIT protein interactions and the downstream effector, MITF-M. J. Pathol. 2004, 202, 463-475. [CrossRef]

25. Uong, A.; Zon, L.I. Melanocytes in Development and Cancer. J. Cell Physiol. 2010, 222, 38-41. [CrossRef] [PubMed]

26. Kang, H.Y.; Suzuki, I.; Lee, D.J.; Ha, J.; Reiniche, P.; Aubert, J.; Deret, S.; Zugaj, D.; Voegel, J.J.; Ortonne, J.P. Transcriptional Profiling Shows Altered Expression of Wnt Pathway- and Lipid Metabolism-Related Genes as Well as Melanogenesis-Related Genes in Melasma. J. Investig. Dermatol. 2011, 131, 1692-1700. [CrossRef]

27. Chung, B.Y.; Noh, T.K.; Yang, S.H.; Kim, I.H.; Lee, M.W.; Yoon, T.J.; Chang, S.E. Gene Expression Profiling in Melasma in Korean Women. Dermatology 2014, 229, 333-342. [CrossRef]

28. Jung, J.M.; Noh, T.K.; Jo, S.Y.; Kim, S.Y.; Song, Y.; Kim, Y.H.; Chang, S.E. Guanine Deaminase in Human Epidermal Keratinocytes Contributes to Skin Pigmentation. Molecules 2020, 25, 2637. [CrossRef]

29. Lee, A.Y. Recent progress in melasma pathogenesis. Pigment Cell Melanoma Res. 2015, 28, 648-660. [CrossRef]

30. Lee, B.W.; Schwartz, R.A.; Janniger, C.K. Melasma. G. Ital. Dermatol. Venereol. 2017, 152, 36-45. [CrossRef] [PubMed]

31. Passeron, T.; Picardo, M. Melasma, a photoaging disorder. Pigment Cell Melanoma Res. 2018, 31, 461-465. [CrossRef] [PubMed]

32. Kwon, S.H.; Na, J.I.; Choi, J.Y.; Park, K.C. Melasma: Updates and perspectives. Exp. Dermatol. 2019, 28, 704-708. [CrossRef] [PubMed]

33. Rajanala, S.; de Castro Maymone, M.B.; Vashi, N.A. Melasma pathogenesis: A review of the latest research, pathological findings, and investigational therapies. Dermatol. Online. J. 2019, 25, 13030. [CrossRef]

34. Kim, N.H.; Choi, S.H.; Lee, T.R.; Lee, C.H.; Lee, A.Y. Cadherin 11 Involved in Basement Membrane Damage and Dermal Changes in Melasma. Acta Derm. Venereol. 2016, 96, 635-640. [CrossRef]

35. Kim, N.H.; Choi, S.H.; Yi, N.; Lee, T.R.; Lee, A.Y. Arginase-2.; a miR-1299 target.; enhances pigmentation in melasma by reducing melanosomedegradation via senescence-induced autophagy inhibition. Pigment Cell Melanoma Res. 2017, 30, 521-530. [CrossRef]

36. Kim, N.H.; Cheong, K.A.; Lee, T.R.; Lee, A.Y. PDZK1 upregulation in estrogen-related hyperpigmentation in melasma. J. Investig. Dermatol. 2012, 132, 2622-2631. [CrossRef] [PubMed]

37. Yang, L.; Ren, S.; Xu, F.; Ma, Z.; Liu, X.; Wang, L. Recent Advances in the Pharmacological Activities of Dioscin. Biomed. Res. Int. 2019, 14, 5763602. [CrossRef] [PubMed]

38. Toyoda, Y.; Takada, T.; Saito, H.; Hirata, H.; Ota-Kontani, A.; Kobayashi, N.; Tsuchiya, Y.; Suzuki, H. Inhibitory effect of Citrus flavonoids on the in vitro transport activity of human urate transporter 1 (URAT1/SLC22A12), A renal re-absorber of urate. NPJ Sci. Food 2020, 4, 3. [CrossRef]

39. Huang, Y.B.; Lee, K.F.; Huang, C.T.; Tsai, Y.H.; Wu, P.C. The effect of component of cream for topical delivery of hesperetin. Chem. Pharm. Bull. 2010, 58, 611-614. [CrossRef]

40. Murata, K.; Takahashi, K.; Nakamura, H.; Itoh, K.; Matsuda, H. Search for Skin-whitening Agent from Prunus Plants and the Molecular Targets in Melanogenesis Pathway of Active Compounds. Nat. Prod. Commun. 2014, 9, 185-188. [CrossRef]

41. Campos, P.M.; Prudente, A.S.; da Silva Horinouchi, C.D.; Cechinel-Filho, V.; Fávero, G.M.; Cabrini, D.A.; Otuki, M.F. Inhibitory effect of GB-2a (I3-naringenin-II8-eriodictyol) on melanogenesis. J. Ethnopharmacol. 2015, 174, 224-229. [CrossRef]

42. Kim, H.J.; Yonezawa, T.; Teruya, T.; Woo, J.T.; Cha, B.Y. Nobiletin, a polymethoxy flavonoid, reduced endothelin-1 plus SCFinduced pigmentation in human melanocytes. Photochem. Photobiol. 2015, 91, 379-386. [CrossRef] [PubMed]

43. Nishina, A.; Ebina, K.; Ukiya, M.; Fukatsu, M.; Koketsu, M.; Ninomiya, M.; Sato, D.; Kimura, H. Dioscin Derived from Solanum melongena L. "Usukawamarunasu” Attenuates $\alpha$-MSH-Induced Melanogenesis in B16 Murine Melanoma Cells via Downregulation of Phospho-CREB and MITF. J. Food Sci. 2015, 80, H2354-H2359. [CrossRef]

44. Rzepka, Z.; Buszman, E.; Beberok, A.; Wrześniok, D. From tyrosine to melanin: Signaling pathways and factors regulating melanogenesis. Postepy Hig. Med. Dosw. 2016, 70, 695-708. [CrossRef] [PubMed]

45. Pillaiyar, T.; Manickam, M.; Jung, S.H. Recent development of signaling pathways inhibitors of melanogenesis. Cell Signal. 2017, 40, 99-115. [CrossRef]

46. Fu, C.; Chen, J.; Lu, J.; Yi, L.; Tong, X.; Kang, L.; Pei, S.; Ouyang, Y.; Jiang, L.; Ding, Y.; et al. Roles of inflammation factors in melanogenesis. Mol. Med. Rep. 2020, 21, 1421-1430. [CrossRef]

47. Han, H.J.; Lim, M.J.; Lee, Y.J.; Lee, J.H.; Yang, I.S.; Taub, M. Uric acid inhibits renal proximal tubule cell proliferation via at least two signaling pathways involving PKC, MAPK, cPLA2, and NF-kappaB. Am. J. Physiol. Renal Physiol. 2007, 292 , F373-F381. [CrossRef]

48. Tang, L.; Xu, Y.; Wei, Y.; He, X. Uric acid induces the expression of TNF- $\alpha$ via the ROS-MAPK-NF- $k B$ signaling pathway in rat vascular smooth muscle cells. Mol. Med. Rep. 2017, 16, 6928-6933. [CrossRef] [PubMed]

49. Doğru, S.; Yaşar, E.; Yeşilkaya, A. Uric acid can enhance MAPK pathway-mediated proliferation in rat primary vascular smooth muscle cells via controlling of mitochondria and caspase-dependent cell death. J. Recept. Signal Transduct. Res. 2021, 1-9. [CrossRef] 
50. Cario, M.; Taieb, A. Isolation and Culture of Epidermal Melanocytes. Methods Mol. Biol. 2019, 1993, 33-46. [PubMed]

51. Ścieżyńska, A.; Nogowska, A.; Sikorska, M.; Konys, J.; Karpińska, A.; Komorowski, M.; Ołdak, M.; Malejczyk, J. Isolation and culture of human primary keratinocytes-a methods review. Exp. Dermatol. 2019, 28, 107-112. [CrossRef] [PubMed] 